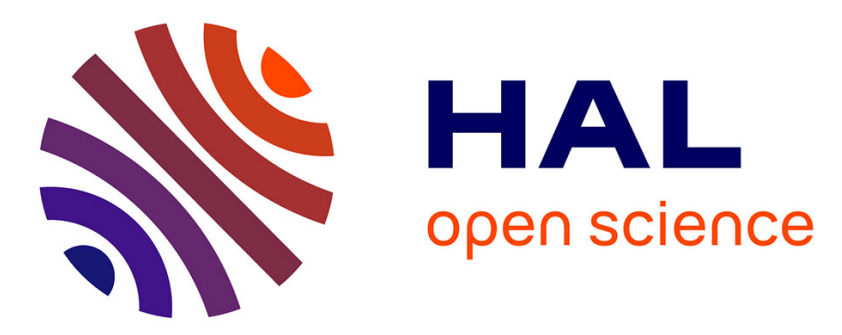

\title{
Recent Developments to Understand Molecular Changes in Shocked Energetic Materials

\author{
Y. Gupta
}

\section{To cite this version:}

Y. Gupta. Recent Developments to Understand Molecular Changes in Shocked Energetic Materials. Journal de Physique IV Proceedings, 1995, 05 (C4), pp.C4-345-C4-356. 10.1051/jp4:1995427 . jpa00253732

\section{HAL Id: jpa-00253732 \\ https://hal.science/jpa-00253732}

Submitted on 1 Jan 1995

HAL is a multi-disciplinary open access archive for the deposit and dissemination of scientific research documents, whether they are published or not. The documents may come from teaching and research institutions in France or abroad, or from public or private research centers.
L'archive ouverte pluridisciplinaire HAL, est destinée au dépôt et à la diffusion de documents scientifiques de niveau recherche, publiés ou non, émanant des établissements d'enseignement et de recherche français ou étrangers, des laboratoires publics ou privés. 


\title{
Recent Developments to Understand Molecular Changes in Shocked Energetic Materials
}

\author{
Y.M. Gupta
}

Shock Dynamics Center and Department of Physics, Washington State University, Pullman, Washington 99164-2814, U.S.A.

\section{Comment:}

First of all I would like to thank Professor S. Odiot and her colleagues for organizing a productive and enjoyable workshop. The presentations at the workshop covered a broad range of topics which reflects the interdisciplinary character of the field. Given my current interests, I shall confine my comments to fundamental issues related to the shock initiation problem. I believe that examination of the shock front remains an important challenge. Without this knowledge, it is difficult to address the question of how the energy is transferred to the molecules. The "up pumping" mechanism proposed by Dlott and Fayer is an interesting hypothesis and one that needs to be examined more carefully. Jerry Dick's ideas about the role of steric hindrance for chemical decomposition merit further study. How fast can this mechanism occur? If a detailed understanding of molecular mechanisms is the need, which it should be, then experiments on single crystals and liquids are essential. These are also the materials for which theoretical calculations can provide insight and, hence, aid experimental analysis. I believe that concerted and sustained efforts on a few selected materials will be most useful. Both experiments and theoretical calculations are needed to understand the role of atomic/molecular defects on a fundamental level. Regarding the onset of chemical reactions, is the problem completely thermal in nature or is it possible to obtain direct electronic excitations? While the latter may not seem energetically favorable at first glance, we need to keep in mind that the shock front represents major disruption of the crystal lattice. These are interesting and difficult questions which will keep us busy for some time.

Once the reaction has been initiated, it is important to follow the various intermediate steps to provide a good link to the reactive flow or the hydrodynamic problem. Experimentally, this is again a very difficult task and anybody who can contribute to this problem should be encouraged to do so. I believe this is an area where high pressure and high temperature studies may provide useful insight.

In closing I make two observations: (i) Understanding the shock response of condensed explosives is a challenging, interdisciplinary problem that will require interactions among experts from several different disciplines. However, care is needed to ensure that scientific convergence will be achieved. (ii) I am very encouraged by the emphasis on fundamental research on energetic materials in France. Thank you for a valuable interaction. 


\section{INTRODUCTION}

A good understanding of molecular mechanisms governing shock induced chemical decomposition in condensed explosives is important to studies of shock sensitivity and the development of new energetic materials. While the need for achieving this understanding has long been recognized, progress has been slow because of the inherent complexity of the scientific issues and the difficulty in conducting the desired shock compression experiments on explosives [1,2]. Historically, the development and use of energetic materials in ordnance applications has relied on advances in formulation and synthesis of energetic materials and correlating these to data from tests designed to measure performance and sensitivity for applications of interest. On the analytic side, studies have focused mainly on the mathematical modeling of phenomena related to the generation, propagation, and stability of detonation waves [1-4].

Plane shock wave experiments provide a well controlled method to examine shock initiation (defined as the onset of measurable chemical decomposition) in condensed energetic materials [5]. Because the loading times in these experiments are comparable to the characteristic times associated with structural and chemical changes, they provide a unique opportunity to examine time dependent microscopic processes associated with these changes. Experimental developments in the United States, the Former Soviet Union, and France in the late sixties and seventies have resulted in time resolved continuum measurements on a large number of explosives $[5,6]$. These data have provided considerable information on the properties of unreacted and reacted explosives. Phenomenological models have been developed to infer details regarding reaction kinetics and energy release rates from stress and/or particle velocity histories. A good account of the macroscopic or continuum mechanisms governing shock sensitivity and the subsequent coupling of chemical decomposition to hydrodynamic flow may be seen in Part Three of Reference 2. In contrast to the considerable progress in continuum measurements and analyses, a good understanding of molecular mechanisms remains a challenging problem primarily due to the lack of real time spectroscopy data in shocked condensed explosives. Some representative examples of the theoretical work on molecular mechanisms governing decomposition and experimental measurements on recovered samples are summarized in Part Two of Reference 2.

The remainder of this paper reviews some recent developments associated with shock induced chemical decomposition by defining the key scientific issues, indicating experimental approaches to time resolved optical spectroscopy, and summarizing some results from our ongoing work on liquid nitromethane. Current limitations of such studies and future directions are indicated. As requested by the workshop organizers, the paper is intended to provide an overview of the developments. More details may be seen in the cited literature.

\section{SCIENTIFIC ISSUES}

Because of the emphasis on microscopic mechanisms, the present discussion is restricted to energetic materials that are macroscopically homogenous. We consider the propagation of a plane shock 
wave in a homogenous explosive and the subsequent build up of pressure due to the initiation of a chemical reaction and the release of chemical energy. It is convenient to consider this problem in three sequential stages:

1. Transfer of energy from the shock wave to the energetic material, that is, "excitation" of the molecules.

2. Initial and subsequent molecular changes associated with chemical decomposition of the "excited" molecules.

3. Significant energy release and coupling of this energy to the propagating shock wave resulting in macroscopically observable pressure increase.

The stages indicated above are an idealization and there will likely be an overlap between these stages depending on the characteristic times associated with each stage. The incorporation of macroscopically heterogeneous materials does not change the problem conceptually; it does introduce an extra complexity at the continuum level because of inhomogenous deformation in the material.

Almost all of the shock wave research on condensed explosives has concentrated on the third stage or the reactive flow problem [1-4]. This emphasis is due primarily to the close relationship of such studies to practical considerations of performance, stability, and safety in various applications. While the benefits of continuum studies on shocked energetic materials are well recognized, it is not possible to get information about the first two stages from the continuum data alone.

Regarding the question of energy transfer, the rise time of the shock front and the nature of atomic/molecular changes at the front are central to understanding this aspect of the problem. As Dremin has pointed out [7], the creators of the ZND model likely envisioned the shock front to be equal to the mean free path of a few molecules and thus their assumption of no reaction in the front seemed reasonable. However, the tremendous rates of loading at the front can significantly alter the material state which in turn influences the subsequent chemical decomposition [7]. For a mechanical disturbance involving the motion of ions or atoms, rise times are expected to be $100 \mathrm{fs}$ or larger. Hence, an examination of how the energy is transferred from the shock wave to molecules in a condensed material requires fast diagnostics. While several competing hypotheses have been put forward [8-10], the energy transfer mechanism remains an open question because of a lack of experimental data. The mechanisms proposed by Faust, and Dlott and Fayer represent a start on this challenging problem [9,10]. Although molecular dynamics calculations are expected to be quite beneficial [11], high time resolution data (ps time scales) are needed to address this issue.

Most of the experimental effort on examining molecular changes has concentrated on the second part, that is, spectroscopic measurements behind the shock front. This is the principal theme of the remainder of this paper. 


\section{OPTICAL SPECTROSCOPY EXPERIMENTS}

The need to understand the shock response of condensed materials at the atomic/molecular level led to the development of optical spectroscopy methods, during the eighties, at several organizations: CEA (France), Livermore, Los Alamos, NRL (Washington, D.C.), Sandia, and our laboratory at Washington State University. These measurements can be divided into two categories: electronic spectroscopy (absorption emission, fluorescence, and reflection) and vibrational spectroscopy (CARS, TRISP, Spontaneous Raman) [12-14]. A noteworthy aspect of the work at Washington State University has been the development of time resolved measurements for both electronic and vibrational spectroscopies. Although time resolved measurements are inherently more difficult, such data are essential for understanding the mechanisms and kinetics that govern time dependent processes such as shock induced chemical decomposition.

During the last fifteen years, time resolved absorption, reflection, fluorescence, and Raman measurements have been obtained on both liquids and solids at Washington State University. Details of our work and spectroscopy efforts by others may be seen in published articles and reviews [12-16]. Here we briefly summarize how the optical spectroscopy experiments are conducted and the current limitations of such measurements.

Optical spectroscopy under shock loading can be considered as a "pump and probe" experiment where the pump is the propagating shock wave. Although there exist many techniques for producing shock waves, flyer plate impact methods provide the best control for producing pulses of known amplitude and duration. Because of the single event nature of the experiment and because the pump is a large amplitude mechanical shock wave produced by impact, these experiments are quite challenging. Figure 1 shows a typical experimental setup that is used for our time resolved spectroscopy measurements in shocked liquid nitromethane [17-19]. In our impact experiments, thin liquid samples (ranging from 10 to $250 \mu \mathrm{m}$ depending on the spectroscopic method) are subjected to step-wise-loading (SWL) due to shock reverberation between two optical windows (typically, sapphire crystals); for a solid sample the front window can be eliminated. By varying the impactor velocity and thickness, shock wave amplitudes and durations can be controlled precisely.

Raman measurements are obtained continuously at pre-determined time intervals (few ns to 50 ns) while the sample is being subjected to shock wave, uniaxial strain loading. Light from a pulsed laser is sent to the sample and scattered light is dispersed in frequency by the spectrometer, temporally dispersed by the electronic streak camera and recorded on a two-dimensional CCD detector; the data consist of intensity-frequency-time output. Other optical components are indicated in the figure; all light transmission to and from the sample is carried out using optical fibers. For destructive experiments, this is an important feature. Using appropriate trigger pins, the pulsed laser and the streak camera are carefully synchronized with both the impact event and the arrival of a the shock wave at the sample. For high time resolution, time synchronization becomes an increasingly difficult and important problem. 
The electronic spectroscopy experiments, particularly reflection and luminescence experiments, are quite similar in setup because both of them utilize a back scattering geometry. The absorption experiment utilizes a single pass transmission measurement and, as such, the impactor arrangement has to be modified to permit light to come from the impactor side [18]. Further details on all of these experimental methods may be seen elsewhere [18-20]. It is worth emphasizing that despite the sophistication of the optical spectroscopy measurements, such data are of limited value unless the continuum parameters (stress amplitude, pulse duration, and temperature when applicable) are precisely known. Hence, the optical measurements compliment the continuum data but are not a substitute for these data. In all of our work, a careful attempt is made to correlate the optical spectroscopy data with continuum results.

Before concluding the brief discussion on optical spectroscopy experiments, a non-trivial issue associated with these measurements needs to be mentioned. Because a shock wave is a propagating disturbance, both spatial and temporal variations need to be considered for the physical quantities of interest. It is for this reason that time resolved continuum measurements using Lagrangian probes (stress and/or particle velocity histories at a fixed material location) have proven immensely beneficial to reactive flow studies [21]. Ideally, one would want the highest spatial and temporal resolution to be achieved simultaneously in the spectroscopy experiments. It is not obvious how such data can be obtained with existing detector technology. In our experiments, we have attempted to meet this requirement by using thin samples $(\sim 10-100 \mu \mathrm{m})$ and recording the spectra with ns resolution. The price we pay is that the sample reaches the peak state through step-wise-loading (SWL) and not through a single shock. By using very thin samples, the total time for shocking up may be reduced considerably but it will still take several steps to reach the peak stress. Alternatively, experiments can be carried out on thick samples to achieve single shock loading but it is not clear that very high spatial and temporal resolution can be achieved for a time-dependent process. Examination of the propagating shock front remains an important challenge.

\section{SHOCK RESPONSE OF NITROMETHANE}

To date, the most comprehensive optical spectroscopy work on a shocked condensed energetic material is the effort on liquid nitromethane at Washington State University [17-19, 22]. Although it is not possible to summarize all aspects of our nitromethane work in a few pages, some features of this work that are important to this workshop are indicated here. Two other examples of comprehensive spectroscopic investigations of shock induced molecular changes may be seen in the Los Alamos work on liquid diatomics [23] (particularly nitrogen) and the work on liquid $\mathrm{CS}_{2}$ at Washington State University [24].

Nitromethane is an insensitive high explosive that is well suited for investigation at an academic institution. It has served as a good prototype material for investigating chemical decomposition in nitrocompounds. Because of these reasons, it is one of the best studied energetic materials and there is a 
large body of spectroscopic data at ambient pressures [25], static high pressure data [26], and continuum data under shock loading [27]. There existed some shock wave spectroscopy data prior to our work but most of these data constituted exploratory efforts and the results were not always in agreement [28-30]. Nitromethane has the additional advantage that it can be sensitized by very small amounts of amines [25]. Potential mechanisms for chemical decomposition in pure and sensitized nitromethane have been considered by Engelke [31], Bardo [32], Constantinou [25], and Cook and Haskins [33]. The Ph.D thesis by Constantinou presents a comprehensive account of thermal decomposition studies in pure and sensitized nitromethane at ambient pressures, and provides an excellent background to the present work [25]. Our objectives in this work were to address the following broad issues:

1. What are the molecular changes associated with shock induced decomposition in nitromethane?

2. Are the differences in pure and sensitized nitromethane response merely differences in threshold pressure and temperature or are the molecular mechanisms themselves different?

3. How are the spectroscopic and continuum studies related?

4. Is the initiation of chemical reaction in nitromethane controlled by pressure or by temperature?

In addition to these issues, there were a number of other needs that were addressed in this work. Among the most prominent of these tasks was the development of an equation of state for liquid nitromethane for use at different initial temperatures. The experimental work on sensitized nitromethane was carried out by $\mathrm{G}$. Pangilinan and $\mathrm{C}$. Constantinou while the pure nitromethane work has comprised the Ph. D thesis of J.M. Winey. Extensive studies [17-19, 22] using both electronic and vibrational spectroscopy on pure and sensitized nitromethane (addition of $0.1 \mathrm{wt}$. percent of ethylenediamine) were conducted. To establish that the data corresponded to irreversible chemical changes, a number of unloading experiments were carried out; in these experiments, the shock pressure is unloaded to very small values while maintaining the sample in a state of uniaxial strain.

The electronic spectroscopy experiments are simpler to carry out and are valuable in establishing threshold conditions for irreversible changes and for examining the kinetics of the decomposition. Winey's measurements on pure nitromethane at different initial temperatures have been very useful in inferring induction times, defined as the time delay between the shock first arriving at the sample and the occurrence of an irreversible change in the electronic spectra [22]. In thermal explosion models, the induction time is related to the reciprocal of the reaction rate. Winey's results demonstrate that, as expected, the absorption data provide a more sensitive probe of the reaction process. This inference is based on the smaller induction time observed in the spectroscopy experiments in contrast to the continuum data. The absorption data from experiments conducted at different initial temperatures show a strong comrelation with the calculated peak temperatures and minimal dependence on pressure [22].

While the electronic spectroscopy measurements are very useful as indicated above, they are difficult to analyze to obtain detailed information about the molecular changes. Hence, we have invested 
a considerable effort in obtaining good time resolved Raman measurements in shocked nitromethane. A recent paper has presented the first evidence of the ability to continuously monitor molecular changes associated with the onset of a chemical reaction in a shocked explosive [19]. In the following paragraphs, we summarize some of the important findings from our Raman measurements $[17,19,22]$. Further details will be presented elsewhere [22,34].

In shocked nitromethane, we are able to monitor several of the intramolecular modes: $\mathrm{CN}$ stretch $\left(917 \mathrm{~cm}^{-1}\right)$, superposed $\mathrm{NO}_{2}$ stretch $/ \mathrm{CH}_{3}$ bend $\left(1400 \mathrm{~cm}^{-1} / 1377 \mathrm{~cm}^{-1}\right)$, and the $\mathrm{CH}_{3}$ stretch $\left(2968 \mathrm{~cm}^{-}\right.$ 1). Studies of unreacted shocked nitromethane showed hardening of different modes and a relative enhancement of the $\mathrm{NO}_{2}$ stretch/CH 3 bend [17]. A representative example of the changes in the Raman spectra in pure nitromethane shocked to $14 \mathrm{GPa}$ is presented in Figure 2. For clarity, not all of the spectra are shown. These data clearly reveal the mode hardening with pressure. As discussed elsewhere $[17,18]$, the response of pure nitromethane is completely reversible to a peak pressure of $14 \mathrm{GPa}$ under step-wise-loading (SWL). As shown in Winey's work, pure nitromethane undergoes irreversible changes at 16-17 GPa and higher pressures, and at higher initial temperatures [22]. The higher peak pressure threshold under SWL, in contrast to single shock loading, is a consequence of the lower peak temperature attained under SWL loading [22].

In contrast to pure nitromethane, our electronic spectroscopy results had shown that sensitized nitromethane ( $0.1 \mathrm{wt}$. percent ethylenediamine) undergoes an irreversible change at peak pressures in the vicinity of $10 \mathrm{GPa}$ (the exact threshold pressure has not been determined) [18]. To investigate the molecular changes associated with the chemical decomposition in sensitized nitromethane, a series of Raman measurements were carried out in sensitized nitromethane [19,34]. Representative spectra of sensitized nitromethane shocked to $14 \mathrm{GPa}$ are presented in Figure 3. Initially, mode hardening and some broadening can be observed in the spectra. The most dramatic result is the difference between the last two spectra which show the complete disappearance of the $\mathrm{CN}$ bond while the other modes, though broadened, are clearly present. These results have been confirmed by higher resolution experiments. Unlike the pure nitromethane spectra at the same shock pressure, the major changes observed in sensitized nitromethane are irreversible.

Our data is consistent with the hypothesis of Cook and Haskins [33] that CN bond scission is important in the shock induced decomposition of amine sensitized nitromethane. They carried out abinitio molecular orbital calculations to show that the activation energy for breaking the $\mathrm{CN}$ bond is lowered significantly because of the interaction of the amino group with the nitromethane molecule. We conclude by pointing out that Winey's results demonstrate that not only is the threshold shock pressure (and temperature) higher for a chemical reaction in pure nitromethane but the reaction mechanism is different [22]. Unlike sensitized nitromethane, $\mathrm{CN}$ bond scission is not the first step in the chemical decomposition of pure nitromethane. To summarize, the presence of amine is not merely a catalyst for the nitromethane reaction; instead, it changes the mechanism of decomposition in shocked nitromethane. 


\section{DISCUSSION AND CONCLUDING REMARKS}

The nitromethane work has demonstrated clearly the ability to monitor molecular changes associated with shock induced chemical decomposition in condensed energetic materials. Despite this exciting development, numerous challenging problems remain both in experimental and theoretical work. Using the nitromethane work as a representative example, some specific issues are discussed first. Next, some broader issues and future needs are commented upon.

Some of the unresolved issues pertaining to the nitromethane work are: temperature measurements in the unreacted material to better define the threshold pressure and temperature conditions and to permit comparisons with more conventional decomposition studies; determining the specific cause of the large background increase (intensity change) and identifying the subsequent reactions and products beyond the initial onset of decomposition; further details about the molecular processes that lead to the lowering of the $\mathrm{CN}$ bond energy because of the presence of the amine. Cook and Haskins [33], and Constantinou [25] have put forward conflicting suggestions as to how this occurs. Resolution to these ideas will require experiments designed to evaluate the different suggestions.

Issues indicated in the preceding paragraph are also expected to arise in similar studies on other materials. Resolution to these issues will require the ability to obtain and analyze intensity measurements with high precision. This is not a simple matter and we are currently conducting an in-depth examination of the response of various components used for signal detection and recording. Given the inherently weak Raman signals, improvements in signal-to-noise ratio remain an ongoing challenge.

At a broader level, we emphasize the need for parallel theoretical studies that can provide guidance to the experimental effort. Given the complexity, cost, and time associated with shock wave experiments, it is not possible to resolve scientific issues by gathering vast amounts of data. Unlike conventional spectroscopy experiments, the spectral and temporal windows have to be pre-determined and current limitations on detectors necessitate a compromise between spectral coverage and spectral resolution. In most of our experiments, we obtain low dispersion measurements (similar to Figures 2 and 3) followed by higher dispersion measurements. Theoretical calculations can significantly increase the efficiency of the experimental work.

An obvious extension of the present work is to examine energetic solids. Dick and coworkers at Los Alamos have drawn interesting correlations between their continuum measurements and optical emission data and suggested a potential micromechanism for shock initiation based on the idea of steric hindrance [35]. Dick has also put forward ideas as to how such a mechanism might work for crystalline nitromethane [36]. We expect to start work in the near future on energetic solids using the electronic and vibrational spectroscopy techniques reported here.

The question of how a shock wave imparts energy to energetic materials is an important one. Dremin has pointed out the need for examining the shock front; this is a problem of long standing both in energetic and non-energetic materials. Given the temporal resolution needs, there is not a simple or 
obvious way to carry out such measurements. We are currently investigating potential experimental approaches that may permit sub-nanosecond time resolution. With regards to the energy transfer problem, molecular dynamics calculations on a realistic system (including incorporation of atomic defects) are expected to be valuable. As stated earlier, the work by Dlott and Fayer provides a comprehensive description of a potential mechanism [10]. Although their hypothesis appears attractive for many situations, it is not clear how their mechanism can explain the observations of Dick and coworkers on crystalline solids [35].

In conclusion, we point out that the subject of shock induced chemical reactions in condensed energetic materials is at an exciting stage. Experimental and theoretical developments have made it possible to address issues that could not be addressed even a few years ago. There exists now the potential for linking molecular mechanisms to continuum and hydrodynamic phenomena. However, the scientific issues of interest are exceedingly complex. In our opinion, careful selection of a limited number of materials and sustained studies on these materials by different groups will be needed to achieve success. Interactions similar to those that took place at this workshop are most beneficial.

\section{ACKNOWLEDGMENTS}

It is a pleasure to acknowledge the hard work and dedication of Gerry Pangilinan, Mike Winey, and Dinos Constantinou who have carried out much of the nitromethane work reported here. George Duvall is sincerely thanked for his work on the nitromethane equation of state and for many helpful discussions. Dave Savage and Kurt Zimmerman contributed to the impact experiments. Finally, this work would not have been possible without the sustained support of the Office of Naval Research; Dr. R.S. Miller is sincerely thanked for his vision, enthusiastic support, and periodic reminders to work on a real explosive.

\section{REFERENCES}

[1] Fickett Ward Davis W.C., Detonation (University of California Press, Los Angeles, 1979).

[2] Cheret R., Detonation of Condensed Explosives (Springer-Verlag, New York, 1993).

[3] Mader C.L., Numerical Modeling of Detonations (University of California Press, Los Angeles, 1979).

[4] Dremin A.N., Lectures on Detonation and Shock Compression of Condensed Materials (Shock Dynamics Center, Washington State University, Pullman, WA 99164-2814, 1993).

[5] Chapter IX of Reference [2].

[6] Proceedings of Sixth through Tenth Symposia (International) on Detonation (Office of the Chief of Naval Research, Arlington, VA. 22217-5000).

[7] Page 59 of Reference [4].

[8] Page 68 of Reference [4]. 
[9] Faust W.L., Science 247 (1989) 37.

[10] Dlott D.D. and Fayer M.D., J. Phys. Chem. 92 (1990) 3798; Tokmakoff A., Fayer M.D., and Dlott D.D., J. Phys. Chem. 97 (1993) 1901.

[11] Brenner D.W., Robertson D.H., Elert M.L., and White C.T., Phys. Rev. Lett. 70 (1993) 2174.

[12] Gupta, Y.M. Shock Compression of Condensed Matter-1991, S.C. Schmidt, R.D. Dick, J.W. Forbes, and D.G. Tasker, Editors (North-Holland, Amsterdam, 1992), 15.

[13] Moore D.S. and Schmidt S.C., Shock Waves in Condensed Matter-1987 (North-Holland, Amsterdam, 1988), 35.

[14] Renlund A.M. and Trott W.M., Shock Waves in Condensed Matter-1987 (North-Holland, Amsterdam, 1988) 547.

[15] Gupta Y.M., High Pressure Research 10 (1992) 713.

[16] Boteler J.M. and Gupta Y.M., Phys. Rev. Lett. 71 (1993) 3497.

[17] Pangilinan G.I. and Gupta Y.M., J. Phys. Chem. 98 (1994) 4522.

[18] Constantinou C.P., Winey J.M., and Gupta Y.M., J. Phys. Chem. 98, (1994) 7767.

[19] Gupta Y.M., Pangilinan G.I., Winey J.M., and Constantinou C.P., Chem. Phys. Lett. 232 (1995) 341.

[20] Gustavsen R. and Gupta Y.M., J. Phys. Chem. 95 (1991) 451.

[21] Cowperthwaite M.C. and Rosenberg J.T., Proceedings of the Sixth Symposium on Detonation (OCNR, Arlington VA, 1976) 786.

[22] Winey J.M., Ph.D. thesis, work in progress.

[23] Schmidt S.C., Moore D.S., and Shaw M.S., Phys. Rev. B 35 (1987) 493.

[24] Yoo C.S., Gupta Y.M., and Duvall G.E., in Shock Compression of Condensed Matter-1989 [North-Holland, Amsterdam, 1990] 675.

[25] Constantinou C.P., The Nitromethane-Amine Interaction, Ph.D. thesis, Cambridge University (1992).

[26] Miller P.J., Black S., and Piermarini G.J., J. Phys. Chem. 93 (1989) 462.

[27] Hardesty D.R., Combust. Flame 27 (1976) 229.

[28] Delpeuch A. and Menil A., Shock Waves in Condensed Matter-1983 (Elsevier Publishing, New York, 1984) 309.

[29] Renlund A.M. and Trott W.M., Shock Compression of Condensed Matter-1989 (Elsevier Publishing, New York, 1990) 875. 
[30] Schmidt S.C., Moore D.S., Shaner J.W., Shampine D.L., and Holt W.T., Physica 139 \& $140 B(1986) 587$.

[31] Engelke R., Schiferl D., Storm C.B., and earl W.L., J. Phys. Chem. 92 (1988) 6815.

[32] Bardo R.D., Int. J. Quantum Chem. 20 (1986) 455.

[33] Cook M.D. and Haskins P.J., Proceedings of the Ninth Symposium (International) on Detonation (OCNR, Arlington, VA. 1989) 1027.

[34] Pangilinan G.I., Constantinou C.P., and Gupta Y.M., manuscript in preparation.

[35] Dick J.J., Mulford R.N., Spencer W.J., Pettit D.R., and Shaw D.C., J. Appl. Phys. 70 (1991) 3572.

[36] Dick J.J., J. Phys. Chem. 97 (1993) 6193.

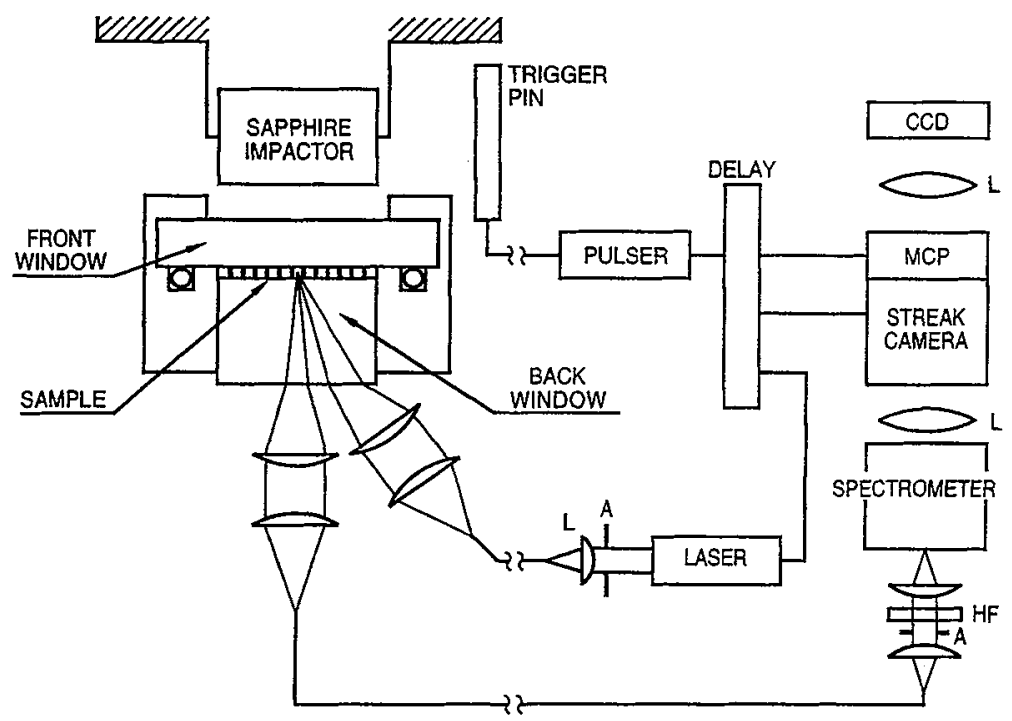

Figure 1. Experimental configuration for obtaining time resolved Raman measurements in shocked nitromethane. L-lens; A-aperture; HF-holographic laser line filter; MCP-microchannel plate intensifier; CCD-charge coupled device detector. Light transmission to and from the sample is carried out using optical fibers. (Ref. 19) 


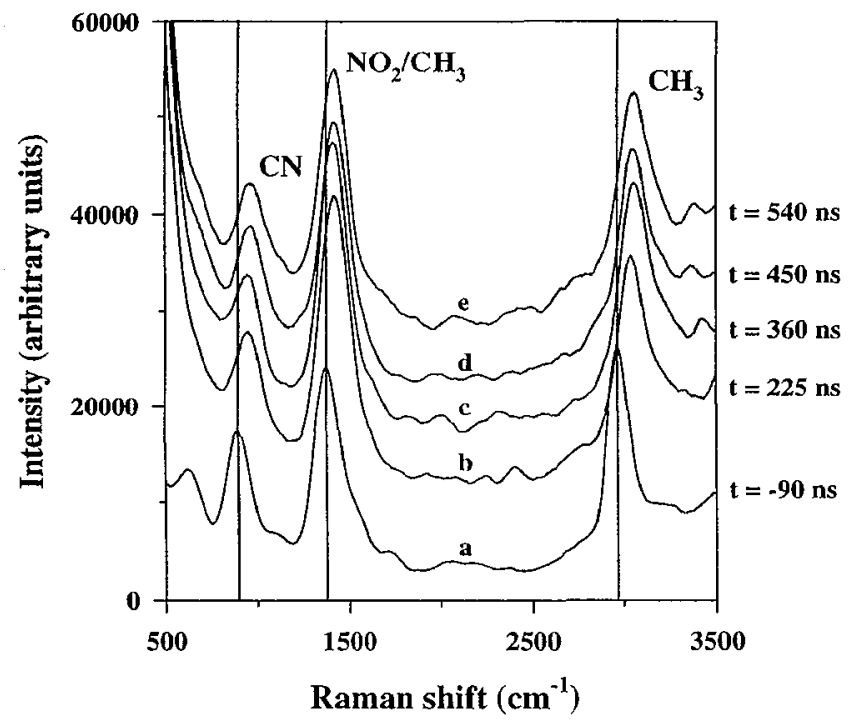

Figure 2. Representative Raman spectra of nitromethane shocked to $14 \mathrm{GPa}$, obtained at (a) ambient pressure; (b) during shock up; (c), (d) and (e) after peak pressure has been reached. $t=0$ corresponds to the time when the shock wave initially enters the sample. Vertical lines mark the frequency positions of the various modes at ambient pressure. For clarity, spectra (b) to (e) are vertically offset successively to 5000 units. (Ref. 19)

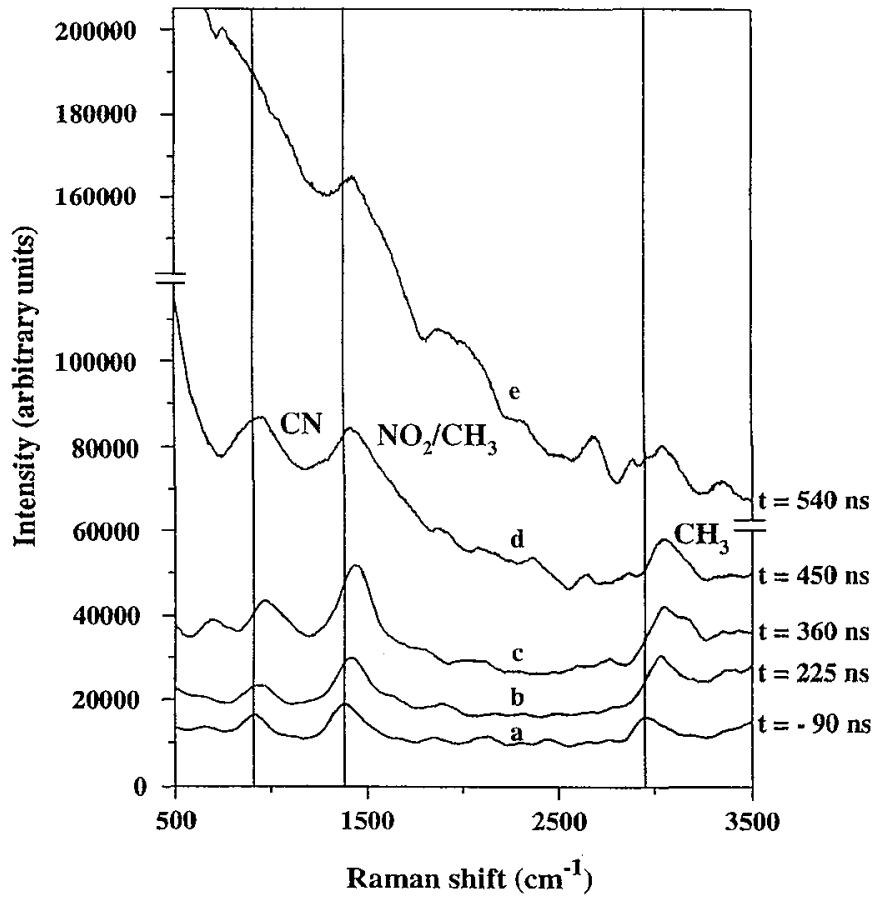

Figure 3. Representative Raman spectra of a solution of nitromethane with ethylenediamine additive $(0.1$ weight $\%$ ) shocked to $14 \mathrm{GPa}$, obtained at times similar to those in Figure 2. Vertical lines mark the frequency positions of the various modes at ambient pressure. Spectra (b) to (e) are vertically offset successively by 5000 units. The $\mathrm{CN}$ vibration softens in spectrum (d) and then disappears in spectrum (e). An increase in the background, due to weak broad features and elastic scattering, is observed after peak pressure is attained. (Ref. 19) 


\section{GENERAL DISCUSSION}

Chairman : Alain Delpuech, Centre d'Etudes du Ripault (CEA/DAM)

Après une première partie consacrée à une approche macroscopique de la détonation, un ensemble de travaux présentés dans cette partie et les suivantes se préoccupent de l'échelle microscopique.

Ces travaux ont souvent pour dénominateur commun un souci de proposer à l'échelle moléculaire une approche qui permette de rendre compte des grandeurs et propriétés caractéristiques de la substance (vitesse de détonation, sensibilité, thermostabilité, ...). Et dans tous les cas ils se concluent par la proposition d'un modèle plus ou moins élaboré faisant appel à un certain nombre de propositions bâties au moyen de théories souvent originales, toujours complexes mais parfois éloignées de la réalité physique des phénomènes mis en cause dans le cas de l'explosif.

La question majeure est de valider ces modeles, de les compléter ou de les infirmer sur la base de résultats expérimentaux.

L'objet de cette table ronde est de confronter les mécanismes de décomposition envisagés d'un point de vue théorique, aux résultats de l'expérience.

Sachant que nous avons tous à l'esprit que ces expériences sont difficiles compte-tenu des contraintes de temps (phénomènes mis en jeu dans des plages de $10^{-12}$ à $10^{-9} \mathrm{~s}$ ), des contraintes de température (plusieurs centaines à milliers de degrés) et de pression (plusiers centaines de kilobars), nous verrons en particulier combien en raison de ces contraintes, le développement de ces expériences doit être spécifique au sujet traité.

Nous nous poserons sans doute la question de savoir si les résultats obtenus à l'aide des techniques spectroscopiques les plus sophistiquées et les plus récentes (picosecondes) sont représentatifs de ce qui se passe dans un front de choc et dans quelle mesure l'utilisation ou l'évolution de ces techniques doivent être analysées.

Ayons bien conscience que cette évolution sera, si elle est possible, très longue, et qu'il serait très dangereux de prendre pour argent comptant les résultats obtenus dans des conditions "tranquilles" et de s'en servir mal à propos pour étayer des modèles rendant compte de phénomènes survenus au moment de la décomposition explosive. 


\title{
4-1 Statements from Laboratories on the Experimental Assessment of the Molecular Mechanisms
}

\author{
A Raman Spectroscopy Study of Nitromethane up to $350^{\circ} \mathrm{C}$ and $35 \mathrm{GPa}$; \\ S.Courtecuisse, F.Cansell, D.Fabre and J.P.Petitet
}

Sub-Picosecond Time-Resolved Spectroscopy of Energetic Materials; The Nitromethane and Nitro-Stilbenes; C.Rajchenbach, G.Jonusauskas and C.Rullì̀re

Experimental Study of Photon-Phonon Interactions in an Explosive by Laser Probe Mass Spectrography ; J.F.Eloy and A.Delpuech

Influence of Initiation Strength, Ambient Inert Gas, Al-Content and Polymeric Binder on the Detonation Products of High Explosives ; F.Volk

Sulphuric Acid Influence on the Nitrocompounds Detonation Reactions ; V.N.Gamezo, B.N.Kondrikov, G.D.Kozac and S.M.Khoroshev

Carbon in Detonation products. A "Three-Phase" Modelisation ; M.L.Turkel and F.Charlet

Rearrangements of Ammonium Nitrate Cluster Ions with High Internal Energy ; R.J. Doyle and Brett I. Dunlap 